\title{
A high-performance interface for grounded conductivity sensors
}

\author{
Xiujun Li and Gerard C M Meijer \\ Faculty of Information Technology and Systems, Delft University of Technology, Mekelweg 4, \\ 2628 CD Delft, The Netherlands \\ E-mail: X.Li@tudelft.nl
}

Received 7 December 2007, in final form 7 August 2008

Published 17 September 2008

Online at stacks.iop.org/MST/19/115202

\begin{abstract}
This paper presents a high-performance interface for grounded conductivity sensors. The interface mainly consists of a sensor driver, an analog front-end, a multiplexer and a voltage-to-time converter. The sensor driver and analog front-end provide a controlled excitation voltage for the sensor and convert the sensor signal (conductance) into a voltage signal. The voltage-to-time converter acts as an asynchronous converter that employs a relaxation oscillator to convert the sensor signals (voltages) into a period-modulated output voltage. The analysis and experiments are performed to optimize the interface circuit with respect to the range of measurable conductance. With a prototype, over a wide conductance range, from $0.01 \mu \mathrm{S}$ to $1 \mathrm{mS}$, the experimental results show random errors with a standard deviation of less than $5.6 \mathrm{nS}$ for a measurement time of $160 \mathrm{~ms}$, and a systematic error of less than $22 \mathrm{nS}$.
\end{abstract}

Keywords: sensor interface, oscillators, grounded conductance measurement

\section{Introduction}

Conductivity sensors are used in various measurement and control systems, for instance, in applications for measuring the properties of biological and chemical materials, for monitoring the environment, for control purposes in chemical processes and for agriculture and food production [1-5]. Thus, performing an accurate and reliable measurement of the conductance is very important for applying the conductivity sensors. In some applications, the system allows the conductivity sensor to be galvanically floating with respect to the interface circuit. In this case, the interface designer is free to connect the sensor that does not disturb biasing of the interface. However, due to safety reasons and/or operating limitations of floating conductivity sensors, in some applications conductivity sensors have to be grounded [6, 7]. When the sensors are in galvanic contact with a conductive liquid, polarization and electro-chemical effects can easily cause electrode corrosion. In order to reduce these effects, the fact that the excitation signal contains a dc component should be avoided.

An important feature of the conductance measurement is its dynamic measurement range. Generally, the range of measured conductivity is very wide, for instance, from
$0.05 \mu \mathrm{S} \mathrm{cm}^{-1}$ for pure water to $55 \mathrm{mS} \mathrm{cm} \mathrm{cm}^{-1}$ for ocean water. By selecting conductivity sensors with different cell constants, the measurement range of the conductance can be optimized. For our design, a conductance measurement range of $0.5 \mu \mathrm{S}$ to $10 \mathrm{mS}$ has been targeted, which could cover a broad range of applications. Regarding the resolution, our design target is $0.05 \mu \mathrm{S}$.

This paper presents the design of a low-cost and accurate interface for grounded conductivity sensors. In this interface, many advanced techniques are applied, such as the chopping technique that reduces the dc offset and low-frequency noise of the electronics, and the auto-calibration technique that ensures high reliability of the measurement system. The interface is optimized with respect to the range of measurable conductance.

The proposed interface is suitable to be applied for both two-electrode and four-electrode conductivity sensors.

\section{The interface system}

\subsection{Basic principles of conductivity sensor and measurement}

Generally, the conductivity sensor is equipped with four or two electrodes. The four-electrode conductivity sensor, which 


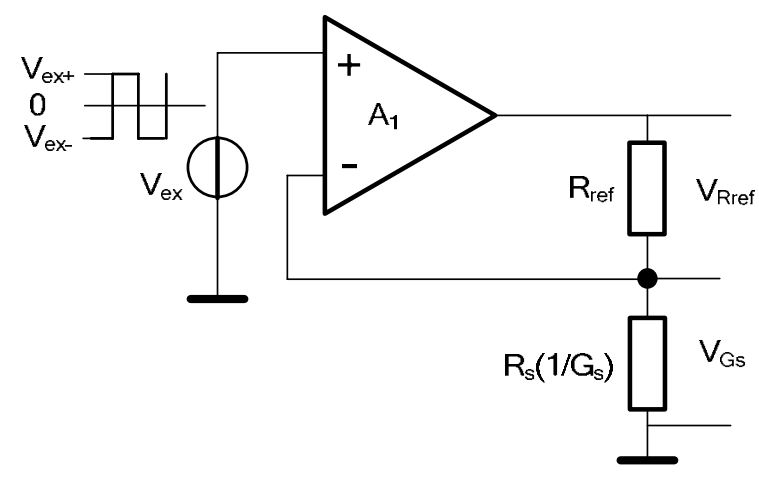

Figure 1. The basic principle of the driving circuit for the measurement of the grounded conductivity sensors.

reduces the effect of the connection wires, is mainly used to measure high conductance values. For lower conductance values the simpler two-electrode sensor can be used. For actual conductivity sensors, its measurement is affected by many nonidealities, including contact potentials, contact impedances and temperature drift. The magnitudes of these effects depend on the materials of the electrode and the object. Generally, they are not stable. Therefore, for an accurate and reliable measurement, the influence of these parasitics should be eliminated or significantly reduced.

The effects of dc contact potentials on the measurement of a conductance $G_{\mathrm{S}}$ can be eliminated by using an ac excitation signal for the sensor. To eliminate the effect of the contact impedances as well as the effect of the lead-wire resistance, a four-wire measurement is applied.

As shown in $[8,9]$, a floating conductance $G_{\mathrm{S}}$ can be measured using an ac voltage excitation $V_{\mathrm{ex}}$. In contrast to this, the circuit presented in this paper should be designed for the measurement of grounded conductances. The driving circuit (figure 1) has been designed just for this application. In this circuit, by the feedback loop around the amplifier $A_{1}$, the voltage $V_{\mathrm{Gs}}$ over the conductance is fixed to the value of $V_{\mathrm{ex}}$. In the case of ideal amplifiers that have a very high loop gain, a negligible input current and an offset voltage, the voltage $V_{\mathrm{Gs}}$ over the conductance $G_{\mathrm{S}}$ equals $V_{\mathrm{ex}}$. The current flow
$I_{\mathrm{Gs}}$ equals the current through $R_{\text {ref }}$. Therefore, the measured conductance equals

$$
G_{\mathrm{S}}=\frac{I_{\mathrm{Gs}}}{V_{\mathrm{Gs}}}=\frac{V_{\mathrm{Rref}}}{V_{\mathrm{ex}}} \frac{1}{R_{\mathrm{ref}}} .
$$

Equation (1) shows that from the measured values of the voltages $V_{\text {ex }}$ and $V_{\text {Rref }}$ the conductance can be calculated.

\subsection{A circuit diagram of the interface}

Figure 2 shows a simplified schematic diagram of the proposed interface, which mainly consists of a sensor driver, an analog front-end, a multiplexer and a voltage-to-time converter.

As shown in the above section, the amplifier AMP1 is employed to establish a controlled excitation voltage $V_{\mathrm{Gs}}$ across the measured conductance $G_{\mathrm{S}}$. The voltage $V_{\mathrm{Gs}}$ is equal to the voltage $V_{\text {ex }}$. To avoid electrolysis, this voltage should be bipolar and its dc component less than the free corrosion potential. The current flow $I_{\mathrm{Gs}}$, which is equal to the current through the resistor $R_{\mathrm{re}}$, is measured by measuring the voltage $V_{\text {Rref }}$ across the resistor $R_{\text {ref. }}$. Both voltages $V_{\text {ex }}$ and $V_{\text {Rref }}$ are sequentially measured using the voltage-to-time converter.

The voltage-to-time converter linearly converts the sensor signal (voltage) and the reference signal (voltage) into periodmodulated signals by employing a relaxation oscillator. The relaxation oscillator is implemented with an amplifier (AMP2), a comparator (COMP), two inverters (IV), two capacitors ( $C_{\text {off }}$ and $\left.C_{\mathrm{int}}\right)$ and a resistor $R_{\mathrm{int}}$. The details of this relaxation oscillator and its performance have been described in $[11,12]$. The capacitor $C_{\mathrm{s}}$ is used to convert the output voltage of the AMP1 into the charge. The multiplexer MUX (figure 2) selects the signal to be measured, $V_{\text {ex }}, V_{\text {Rref }}, V_{\text {off,Rref }}$ or $V_{\text {off,Gx }}$, where $V_{\text {off,Rref }}$ and $V_{\text {off,Gx }}$ are the offset measurements at the corresponding $\mathrm{CM}$ voltage levels. The capacitor $C_{\mathrm{s}}$ samples one of these four voltage signals.

With the relaxation oscillator, an ac square-wave excitation signal $V_{\mathrm{ex}}$ is generated. The use of ac signals is important to reduce the electro-chemical effects and the effects of dc drift and parasitic Seebeck voltages. Meanwhile, the ac square-wave excitation signal is also used to implement

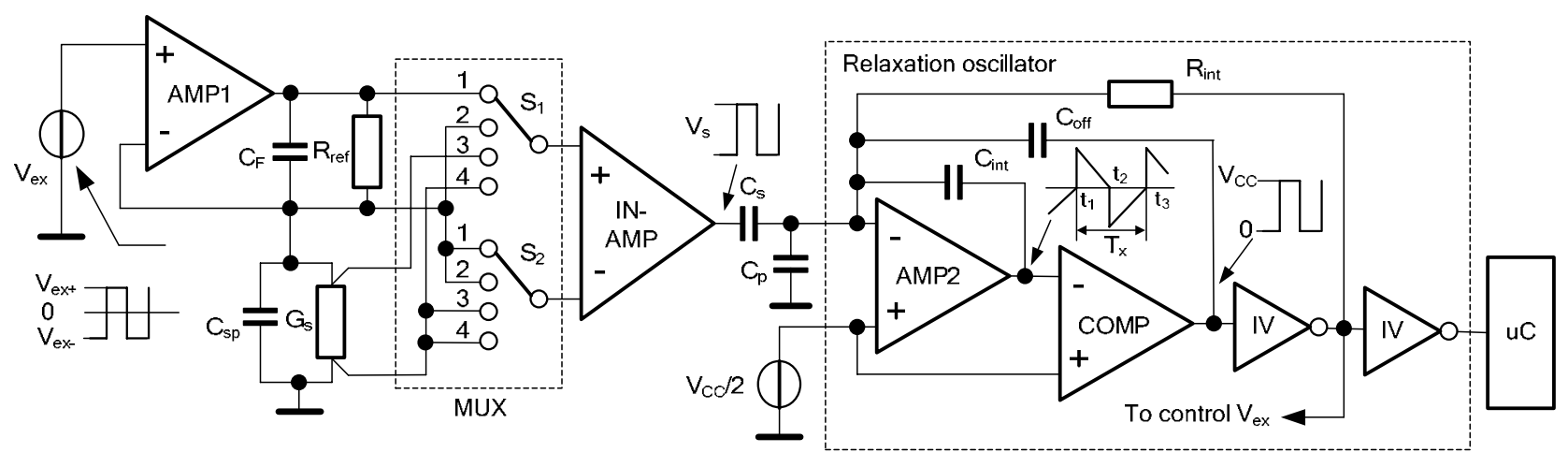

Figure 2. A simplified interface for the grounded conductance measurement. 
the chopping technique $[9,10]$, synchronized with voltageto-time conversion. The chopping technique is implemented in the following way: when $V_{\text {ex }}$ makes an up-going step, a positive charge $V_{\mathrm{s}} C_{\mathrm{s}}$ is converted into a time interval and when $V_{\mathrm{ex}}$ makes a down-going step, a negative charge $-V_{\mathrm{s}} C_{\mathrm{s}}$ is converted into a time interval. The sum of these two time intervals composes a period of the oscillator signal. The chopping technique reduces the effects of offset, $1 / f$ noise and low-frequency interference, significantly. This enables the use of low-cost CMOS technology for accurate measurement systems.

The offset capacitor $C_{\text {off }}$ is used to bring the oscillator frequency within its linear range. The capacitor $C_{\mathrm{p}}$ represents the parasitic capacitance including the connection-wire's and component's capacitances. This capacitance will affect the noise performance of the interface (see section 3 ).

As presented in $[11,12]$, the period of the relaxation oscillator equals

$T=4 R_{\mathrm{int}} \frac{V_{\mathrm{s}} C_{\mathrm{s}}+V_{\mathrm{CC}} C_{\mathrm{off}}}{V_{\mathrm{CC}}}+\tau=4 R_{\mathrm{int}} C_{\mathrm{s}} \frac{V_{\mathrm{s}}}{V_{\mathrm{CC}}}+4 R_{\mathrm{int}} C_{\mathrm{off}}+\tau$,

where $\tau$ is the delay time of the oscillating loop. From this relation, it can be found that once $R_{\text {int }}$ is defined $C_{\text {off }}$ will determine the maximum frequency of the oscillator and that $C_{\mathrm{s}}$ will determine the dynamic range of the oscillator.

During the measurement phases of $V_{\mathrm{ex}}, V_{\mathrm{Rref}}, V_{\mathrm{off}, \mathrm{ref}}$ and $V_{\text {off,Gs }}$, the period times of the output signal of the oscillator amount to $T_{\mathrm{VGs}}, T_{\mathrm{IGs}}, T_{\mathrm{off}, \mathrm{Gs}}$ and $T_{\mathrm{off}, \text { ref, }}$, respectively. These period times amount to

$$
\begin{gathered}
T_{\mathrm{VGs}}=4 R_{\mathrm{int}} C_{\mathrm{s}} \frac{V_{\mathrm{ex}}}{V_{\mathrm{CC}}}+\left(4 R_{\mathrm{int}} C_{\mathrm{off}}+\tau_{1}\right) \\
T_{\mathrm{IGs}}=4 R_{\mathrm{int}} C_{\mathrm{s}} \frac{V_{\mathrm{Rref}}}{V_{\mathrm{CC}}}+\left(4 R_{\mathrm{int}} C_{\mathrm{off}}+\tau_{2}\right) \\
T_{\mathrm{off}, \mathrm{Gs}}=4 R_{\mathrm{int}} C_{\mathrm{off}}+\tau_{1} \\
T_{\mathrm{off}, \mathrm{ref}}=4 R_{\mathrm{int}} C_{\mathrm{off}}+\tau_{2},
\end{gathered}
$$

where $\tau_{1}$ and $\tau_{2}$ represent the additive errors including those which depend on the common-mode voltage. From equations (1)-(3), for the conductance $G_{\mathrm{S}}$ it can be found that

$$
G_{\mathrm{S}}=\frac{T_{\mathrm{IGs}}-T_{\mathrm{off}, \mathrm{ref}}}{T_{\mathrm{VGs}}-T_{\mathrm{off}, \mathrm{Gs}}} \cdot \frac{1}{R_{\mathrm{ref}}} .
$$

This result does not depend on the unknown parameters of the interface, $R_{\mathrm{int}}, V_{\mathrm{CC}}, C_{\mathrm{s}}, C_{\mathrm{off}}, \tau_{1}$ and $\tau_{2}$. In this way, the interface is auto-calibrated for additive or multiplicative errors. Even in the case of slow variations of all these parameters, their effects are eliminated. Also, the effects of the input offset voltage and biasing current of amplifiers are eliminated. The algorithm can be implemented using, for instance, a microcontroller.

\section{Non-idealities}

As described in section 2, many unknown parameters of the interface are eliminated by means of auto-calibration (equation (4)). Also, the use of this auto-calibration will eliminate the effects of many non-idealities of the amplifier
(AMP2) and comparator (COMP). For instance, the input offset voltages of the amplifier and comparator will induce an offset error on the oscillator period. The biasing current of the amplifier and the asymmetry of the comparator output with respect to $V_{\mathrm{CC}} / 2$ will induce a multiplicative error in the oscillator period. These multiplicative and offset errors will be eliminated by the use of auto-calibration. However, some effects cannot be eliminated by this technique and should be taken into account during the interface design.

\subsection{Noise}

For the conductance measurement with the interface shown in figure 2, the noise originates mainly from three parts: the oscillator thermal noise, amplifier noise (AMP1 and INAMP) and the quantization noise caused by sampling in the microcontroller. As compared to the amplifier noise, the thermal noise of the reference resistor (in our case $R_{\text {ref }}<1$ $\mathrm{k} \Omega$ ) is negligible. Now, the relative errors caused by these three noise sources will be analyzed.

3.1.1. Oscillator thermal noise. As described in [11, 13], the oscillator thermal noise is mainly determined by the noise properties of the integrator amplifier and comparator. When the comparator is implemented with an opamp with a limited bandwidth, then its noise contribution can be neglected. The oscillator noise is mainly due to the noise of the amplifier AMP2 (figure 2) and is affected by the integrator bandwidth and the measurement time. As discussed in [11, 13], the normalized noise error of the oscillator is defined as

$$
\begin{aligned}
\zeta & =\frac{\Delta T_{x}}{T_{x}}=\frac{t_{n 1}+t_{n 2}}{T_{x}} \\
& =\frac{C_{\text {int }}}{2\left(V_{\mathrm{CC}} C_{\text {off }}+V_{\mathrm{s}} C_{\mathrm{s}}\right)}\left(2 v_{n}\left(t_{2}\right)-v_{n}\left(t_{1}\right)-v_{n}\left(t_{3}\right)\right),
\end{aligned}
$$

where $v_{n}\left(t_{1}\right), v_{n}\left(t_{2}\right)$ and $v_{n}\left(t_{2}\right)$ are the noise voltages at the input of the comparator at the switching moments $t_{1}, t_{2}$ and $t_{3}$ (indicated in figure 2), respectively. The standard deviation $\sigma_{\text {nosc }}$ of (5) is given by

$$
\sigma_{\text {nosc }}=\sqrt{\int_{0}^{B_{\mathrm{eq}}}\left|H_{v}(\mathrm{j} f)\right|^{2} S_{v}(f) \mathrm{d} f},
$$

where $S_{v}(f)$ is the power spectral density (PSD) of the input noise $v_{n}$ and $B_{\text {eq }}$ is the equivalent bandwidth of the system. For one sinusoidal component of the input noise, in the frequency domain the transfer function $\left|H_{v}(\mathrm{j} f)\right|$ is given by [11]

$$
\left|H_{v}(\mathrm{j} f)\right|=\frac{2 C_{\mathrm{int}}}{\left(V_{\mathrm{CC}} C_{\mathrm{off}}+V_{\mathrm{s}} C_{\mathrm{s}}\right)} \sin ^{2}\left(\frac{\pi f}{2} T_{x}\right) .
$$

For the input noise voltage $v_{\text {amp2 }}$ of the AMP2, its equivalent $\mathrm{PSD}$ at the input of the comparator is given by [11]

$$
S_{v}(f)=S_{v \mathrm{amp} 2}(f)\left(\frac{C_{\mathrm{off}}+C_{\mathrm{s}}+C_{\mathrm{int}}+C_{\mathrm{p}}}{C_{\mathrm{int}}}\right)^{2},
$$

where $S_{v a m p 2}$ is the power spectral density (PSD) of the input noise $v_{\mathrm{amp} 2}$ of the AMP2 and $C_{\mathrm{p}}$ is the parasitic capacitance from the inverting input of AMP2 to the ground. 
In our case, the bandwidth $B_{\text {eq }}$ is limited by the bandwidth $f_{\mathrm{T}}$ of amplifier AMP2, which is

$$
B_{\text {eq }}=B_{\text {amp } 2}=\left(\frac{C_{\text {int }}}{C_{\text {off }}+C_{\mathrm{s}}+C_{\text {int }}+C_{\mathrm{p}}}\right) f_{\mathrm{T}} .
$$

From equations (6)-(9), for a single period of the oscillator output, the standard deviation of the oscillator noise $\sigma_{\text {nosc }}$ is found to be

$$
\begin{array}{r}
\sigma_{\text {nosc }, \text { single }}=\frac{C_{\text {int }}}{V_{\mathrm{CC}} C_{\mathrm{off}}+V_{\mathrm{s}} C_{\mathrm{s}}} \\
\times \sqrt{\frac{3}{2} \frac{\left(C_{\mathrm{off}}+C_{\mathrm{s}}+C_{\mathrm{int}}+C_{\mathrm{p}}\right)}{C_{\mathrm{int}}} f_{\mathrm{T}} S_{v \mathrm{amp} 2} .}
\end{array}
$$

For $N(N \gg 1)$ periods of the oscillator output, the standard deviation of the oscillator noise $\sigma_{\text {nosc }}$ equals

$$
\sigma_{\text {nosc }, N}=\frac{\sigma_{\text {nosc, } \text { single }}}{\sqrt{N}} .
$$

So, the standard deviation of the oscillator noise is reduced with the square root of the number of periods $(N)$.

3.1.2. Amplifier noise (AMP1 and IN-AMP). With figure 1 and equation (1), it can be calculated that the noise $\sigma_{\text {namp } 1}$ caused by the noise of the amplifier AMP1 amounts to

$\sigma_{n \mathrm{amp} 1} \approx \sqrt{\frac{v_{\mathrm{amp} 1}^{2}}{V_{\mathrm{ex}}^{2}}\left(1+\frac{V_{\mathrm{ref}}^{2}}{V_{\mathrm{ex}}^{2}}\right)}=\sqrt{\frac{v_{\mathrm{amp} 1}^{2}}{V_{\mathrm{ex}}^{2}}\left(1+R_{\mathrm{ref}}^{2} G_{\mathrm{S}}^{2}\right)}$,

where $v_{\text {amp1 }}$ is the RMS value of the equivalent input noise of AMP1.

A similar result is found for the effect of the noise of amplifier IN-AMP, which has unity gain. When considering a measurement over $N$ periods, the total-noise contribution of both amplifiers, AMP1 and IN-AMP, is given by

$$
\sigma_{n \mathrm{amp}} \approx \sqrt{\frac{v_{\mathrm{amp} 1}^{2}+v_{I N \mathrm{amp}}^{2}}{N V_{\mathrm{ex}}^{2}}\left(1+R_{\mathrm{ref}}^{2} G_{\mathrm{S}}^{2}\right)}
$$

where $v_{\text {INamp }}$ denotes the RMS values of the equivalent input noise of the IN-AMP.

\subsubsection{Quantization noise. Digitizing the period times with} a counter will cause quantization noise. The level of this quantization noise depends on the length of the period time and the counter clock. As described in $[11,13]$, the standard deviation of the relative error $\sigma_{\text {nqt }}$ caused by the quantization noise amounts to

$$
\sigma_{\text {nqt }} \approx \frac{1}{\sqrt{3}} \frac{1}{N T_{\mathrm{p}} f_{\mathrm{c}}},
$$

where $f_{\mathrm{c}}$ is the sampling frequency of the counter and $T_{\mathrm{p}}$ is the period time of the oscillator. The quantization noise is inversely proportional to the measurement time $\left(N T_{\mathrm{p}}\right)$. Equations (11)-(14) show that with increasing $N$, the quantization noise decreases more rapidly than the other two noise effects.

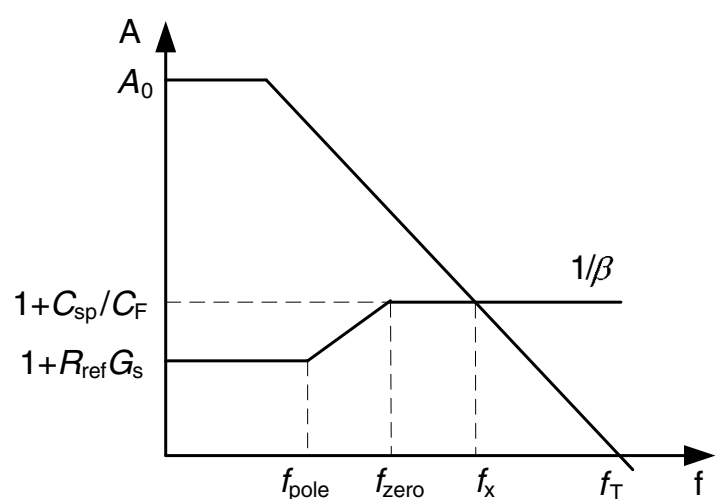

Figure 3. Magnitude Bode plot.

\subsection{The leakage current of the multiplexer}

The leakage current of the multiplexer will induce an additive error on the measured conductance. Only the leakage currents of the switches that are connected to the junction between $R_{\text {ref }}$ and $G_{\mathrm{S}}$ contribute to the measurement error. The leakage currents $I_{S i, j}$ of the four multiplexer switches cause an error $\delta G_{\mathrm{S}}$ in the measurement of $G_{\mathrm{S}}$, which amounts to

$$
\delta G_{\mathrm{S}} \approx-\frac{I_{S 1,2}+I_{S 1,3}+I_{S 2,1}+I_{S 2,2}}{V_{\mathrm{ex}}} .
$$

To reduce this error, a multiplexer with a low leakage current should be used.

\subsection{Parasitic capacitance of the sensor}

Due to the connecting cables, sensor structure and the interface circuit, there exists a parasitic capacitance $C_{\mathrm{sp}}$, which is in parallel with the sensor. This parasitic capacitor will induce a zero in the transfer function of the sensor driver electronics, which will result in the stability problem.

To compensate for this effect, a feedback capacitor $C_{\mathrm{F}}$ is applied in parallel with $R_{\text {ref }}$ (see figure 2). Figure 3 depicts the Bode plot for gain $A$ and $1 / \beta$ of the amplifier AMP1 in figure 2. From figure 2 , the feedback factor $\beta$ is found to be

$$
\beta=\frac{1+\mathrm{s} R_{\mathrm{ref}} C_{\mathrm{F}}}{1+R_{\mathrm{ref}} G_{\mathrm{S}}+\mathrm{s} R_{\mathrm{ref}}\left(C_{\mathrm{F}}+C_{\mathrm{sp}}\right)} .
$$

In this figure, $f_{\mathrm{T}}$ represents the unity-gain bandwidth of AMP1. Furthermore, the frequency values indicated along the horizontal axis are

$$
\begin{gathered}
f_{\text {pole }}=\frac{1+R_{\mathrm{ref}} G_{\mathrm{S}}}{2 \pi R_{\mathrm{ref}}\left(C_{\mathrm{F}}+C_{\mathrm{sp}}\right)} \\
f_{\text {zero }}=\frac{1}{2 \pi R_{\mathrm{ref}} C_{\mathrm{F}}} \\
f_{x}=\frac{f_{T}}{1+C_{\mathrm{sp}} / C_{\mathrm{F}}} .
\end{gathered}
$$

To have a stable sensor driver, the following conditions should be met:

$$
f_{\text {pole }}<f_{x} \quad f_{\text {zero }}<f_{x} .
$$


Then, from equations (17) and (18) the feedback capacitor $C_{F}$ should be selected according to the condition

$$
C_{\mathrm{F}}>\max \left(\frac{1+\sqrt{1+8 \pi R_{\mathrm{ref}} C_{\mathrm{sp}} f_{\mathrm{T}}}}{4 \pi R_{\mathrm{ref}} f_{\mathrm{T}}}, \frac{1+R_{\mathrm{ref}} G_{\mathrm{S}}}{2 \pi R_{\mathrm{ref}} f_{\mathrm{T}}}\right) .
$$

It is shown that a large value of capacitor $C_{\mathrm{F}}$ makes AMP1 more stable. However, a large value of capacitor $C_{\mathrm{F}}$ will also induce a large error in the $G_{\mathrm{S}}$ measurement.

Suppose that the used amplifier (AMP1) has a unity-gain bandwidth $f_{\mathrm{T}} \gg f_{\text {zero }}$ (see equation (17)), as defined in (1); in the frequency domain the measured conductance $G_{\mathrm{sm}}(f)$ is given by

$$
G_{\mathrm{sm}}(f)=G_{\mathrm{S}} \frac{1+\mathrm{s} C_{\mathrm{sp}} / G_{\mathrm{S}}}{1+\mathrm{s} R_{\mathrm{ref}} C_{\mathrm{F}}} .
$$

Then, in the time domain the following result is obtained:

$$
G_{\mathrm{sm}}(t) \approx G_{\mathrm{S}}+\frac{1}{R_{\text {ref }}}\left(\frac{C_{\mathrm{sp}}}{C_{\mathrm{F}}}-R_{\mathrm{ref}} G_{\mathrm{S}}\right) \mathrm{e}^{-\frac{T_{I G S}}{2 R_{\mathrm{ref}} C_{\mathrm{F}}}} .
$$

So the error $\delta G_{\mathrm{S}}$ in the $G_{\mathrm{S}}$ measurement induced by the capacitor $C_{\mathrm{F}}$ amounts to

$$
\delta G_{\mathrm{S}}=G_{\mathrm{sm}}-G_{\mathrm{S}}=\frac{1}{R_{\mathrm{ref}}}\left(\frac{C_{\mathrm{sp}}}{C_{\mathrm{F}}}-R_{\mathrm{ref}} G_{\mathrm{S}}\right) \mathrm{e}^{-\frac{T_{I G s}}{2 R_{\mathrm{ref}} C_{\mathrm{F}}}} .
$$

\subsection{Temperature drift}

As described in section 2, the offset and gain errors of the interface are eliminated by applying auto-calibration (see also equation (4)). Auto-calibration will also eliminate the effects of changes caused by the drift of the offset voltage and interface gain as caused by temperature variations. Yet, the reference resistor $R_{\text {ref }}$ should have a low-temperature coefficient because its temperature performance will directly affect the absolute measurement result of the conductance $G_{\mathrm{S}}$ (see equation (4)).

\section{Range of measurable conductance}

\subsection{The minimum measurable conductance}

The minimum measurable conductance (the resolution) is mainly limited by the noise level of the interface $\sigma_{\mathrm{nG}}$ and the choice of the reference resistor $R_{\text {ref. }}$ The noise of the interface $\sigma_{\mathrm{nG}}$ is mainly composed of the three items mentioned in section 3 . Then, the minimum measurable conductance $G_{x, \min }$ is given by

$$
G_{x, \min } \geqslant \frac{\sigma_{n G}}{R_{\text {ref }}},
$$

which shows that a larger value of $R_{\text {ref }}$ results in a better resolution.

\subsection{The maximum measurable conductance}

The maximum measurable conductance is mainly limited by the output drive current $I_{\mathrm{OUT}}$,max of the amplifier AMP1 and the linear dynamic range of the relaxation oscillator. With respect to $I_{\text {OUT,max }}$, the maximum measurable conductance $G_{x, \max }$ is limited to $G_{x, \max } \leqslant\left(I_{\mathrm{OUT}, \max } / V_{\mathrm{ex}}\right)$.

By reducing the amplitude of the excitation signal, the maximum measurable conductance can be increased.
However, this will increase the contribution of the amplifier noise (AMP1 and IN-AMP) too.

Concerning the linear dynamic range of the relaxation oscillator, the maximum voltage over reference resistor is designed as $V_{\text {Rref,max }}=R_{\text {ref }} I_{\text {OUT,max }}=V_{\text {ex }}$. So that, the maximum measurable conductance $G_{x, \max }$ is given by

$$
G_{x, \max } \leqslant 1 / R_{\text {ref }} .
$$

In our design, the maximum measurable conductance $G_{x, \max }$ is limited to $1 / R_{\text {ref. }}$.

\section{Experimental results}

The described conductivity-sensor interface has been implemented and tested, using the circuit shown in figure 2. For the operational amplifiers, dual OPAMPs (OPA2132PA) have been used. The relaxation oscillator is built with an amplifier (OPA2350), an inverter (HC04), a comparator implemented with an opamp (OPA2132PA) and some passive components. The circuit is powered by a dual power supply of $\pm 5 \mathrm{~V}$. The multiplexer (MAX4559) has been chosen because of its low leakage current. The ac square-wave excitation signal is generated by the oscillator itself. To have the best performance, the values of passive components of the oscillator are selected as $R_{\text {int }}=2 \mathrm{M} \Omega, C_{\text {off }}=6.2 \mathrm{pF}, C_{\mathrm{s}}=$ $82 \mathrm{pF}$ and $C_{\mathrm{int}}=27 \mathrm{pF}$. In this case, the frequency of the excitation signal amounts to $8-20 \mathrm{kHz}$ depending on the value of the measured conductance. A fast microcontroller of the type LPC2101, which has a $70 \mathrm{MHz}$ counting frequency, is employed to measure the output period of the oscillator. It also processes the data and communicates with the outside digital world. Because the performances of the reference resistor directly affect the measurement results, a high-precision type (tolerance: $< \pm 0.01 \%$ and temperature coefficient: $<0.6 \mathrm{ppm}{ }^{\circ} \mathrm{C}^{-1}$ ) has been chosen. The performance of the interface has been tested for the case that the controlled excitation voltage $V_{\text {ex }}$ has a peak-to-peak value of $\pm 200 \mathrm{mV}$. Depending on the electro-chemical properties, the amplitude of the excitation signal can be adjusted for a value less than the free corroding potential.

\subsection{Standard deviation of the noise}

The standard deviation of the interface was measured with $R_{\text {ref }}$ $=1000.06 \Omega$ and $G_{\mathrm{S}}=49.986 \mu \mathrm{S}$, and was calculated with 500 measurement samples. The measurement standard deviation has also been determined as a function of the measurement time, which is the total time for the measurement of $T_{\mathrm{VGs}}$, $T_{\text {IGs }}, T_{\text {off,Gs }}$ and $T_{\text {off,ref. }}$ This measurement result is depicted in figure 4. As an example, for a measurement time of $160 \mathrm{~ms}$, the measured standard deviation amounts to $5.6 \mathrm{nS}$ for the range of $0.01 \mu \mathrm{S}$ to $1 \mathrm{mS}$.

For very short measurement times, the quantization noise, which originates from the sampling by the microcontroller, is dominant. For these short times, the resolution is inversely proportional to the measurement time. For longer measurement time, the interface thermal noise is dominant, which is inversely proportional to the square root of the 


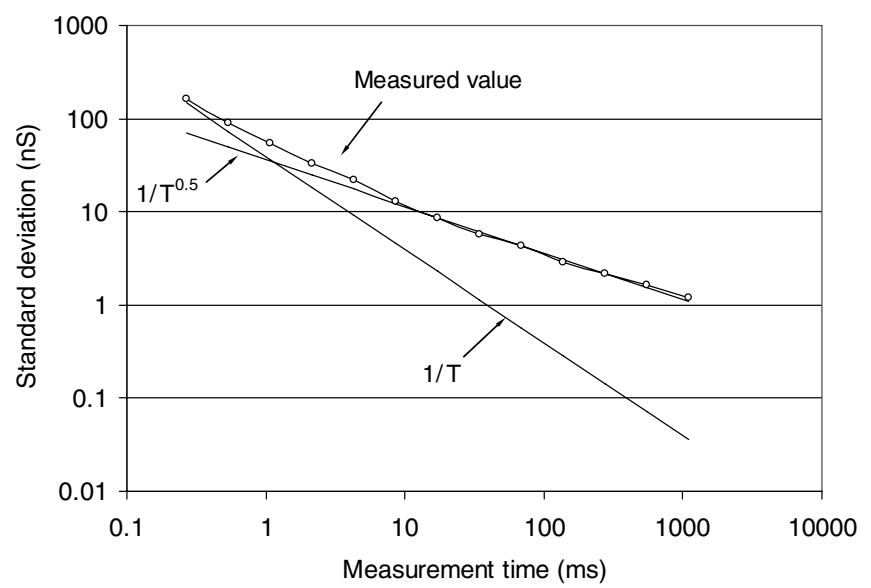

Figure 4. The measured standard deviation.

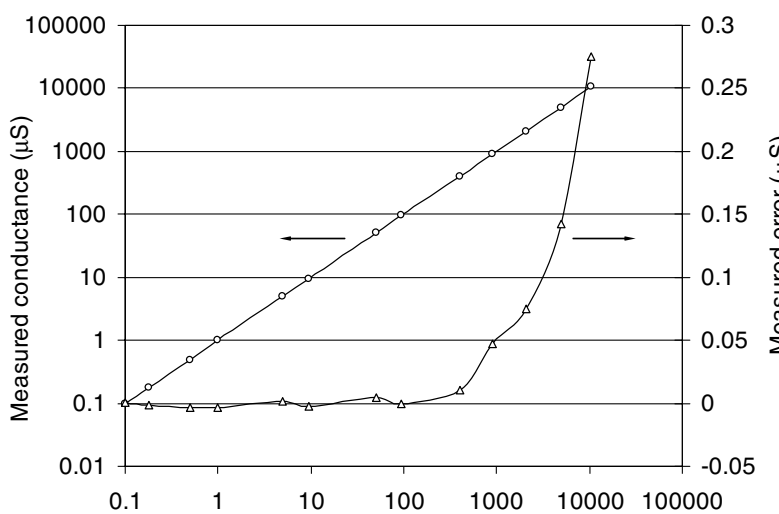

(a) Conductance to be measured $(\mu \mathrm{S})$

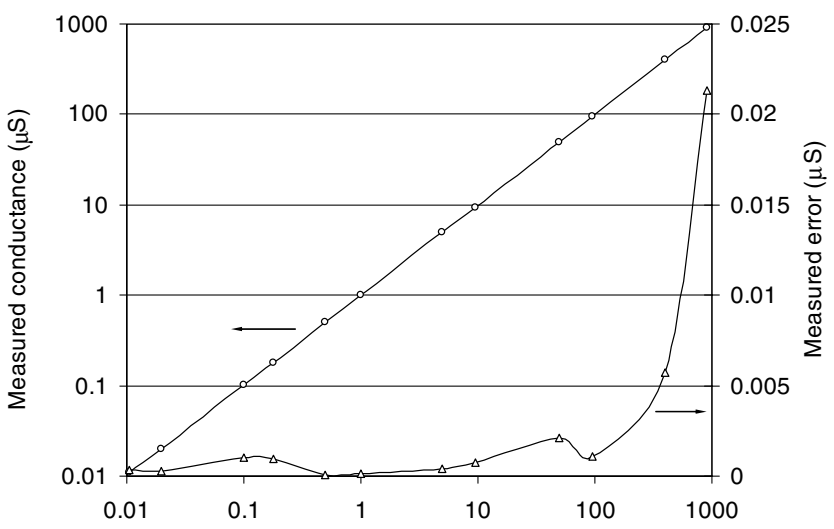

(b) Conductance to be measured $(\mu \mathrm{S})$

Figure 5. Systematic errors with $(a) R_{\text {ref }}=104.065 \Omega$ and $(b) R_{\text {ref }}=$ $1000.06 \Omega$.

measurement time. A longer measurement time results in a lower noise level, therefore a smaller measurable conductance.

\subsection{The systematic error}

The systematic error of the interface was measured with a measurement time of $160 \mathrm{~ms}$ and two different referenceresistance values (104.065 $\Omega$ and $1000.06 \Omega)$. The measurement results for the conductance $G_{\mathrm{s}}$ are depicted in figure 5 .

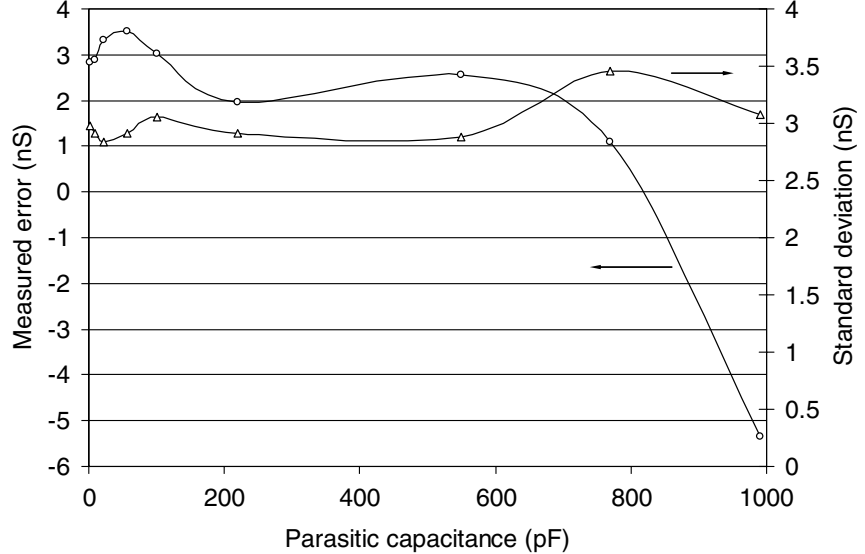

Figure 6. The effect of the parasitic capacitance $C_{\mathrm{sp}}$ for the systematic error and the standard deviation.

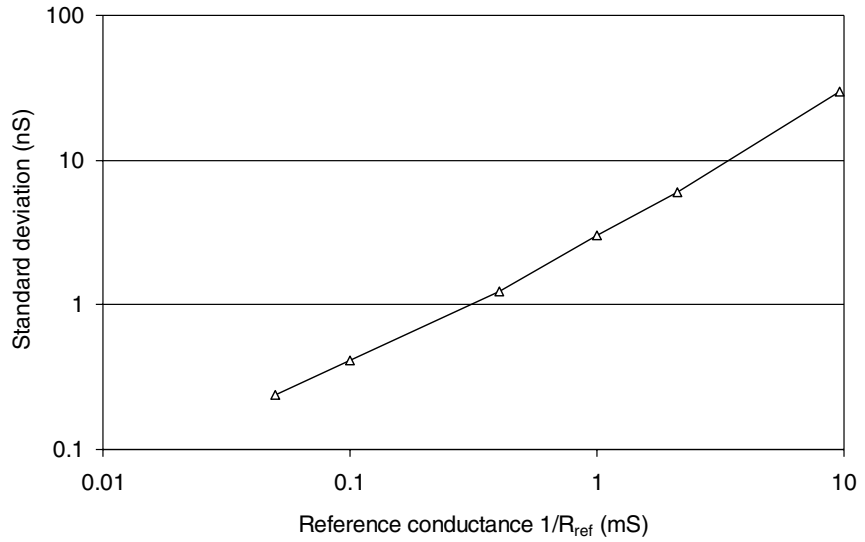

Figure 7. The effect of the reference resistor.

It is shown that the minimum measurable range is enlarged by increasing the value of $R_{\text {ref. }}$ For high values of the conductance, as depicted at the right-hand side of the graph, the measured error increases linearly with the measured conductance. It should be noted that it concerns a linear error, which has been depicted on a logarithmic scale. The maximum errors amount to $0.28 \mu \mathrm{S}$ and $0.022 \mu \mathrm{S}$ for the measurement ranges of $0.1 \mu \mathrm{S}$ to $10 \mathrm{mS}$ and $0.01 \mu \mathrm{S}$ to $1 \mathrm{mS}$, respectively. These errors could be due to the measurement error of the reference resistance: an error similar to that shown in figure 5 can be caused by an error of only $0.0025 \%$ in the value of the reference resistance.

\subsection{The effect of the parasitic capacitance}

The effects of the parasitic capacitor $C_{\mathrm{sp}}$ on the interface accuracy were measured for $C_{\mathrm{F}}=1 \mathrm{nF}, R_{\text {ref }}=1000.06 \Omega$ and $G_{\mathrm{S}}=49.986 \mu \mathrm{S}$. The results are shown in figure 6 . It is shown that up to $C_{\mathrm{sp}}=1 \mathrm{nF}$ the measurement error is less than $6 \mathrm{nS}$. When the measured conductance $G_{\mathrm{S}}$ is reduced to, for instance, $1 \mu \mathrm{S}$, the parasitic-capacitance effect on the standard deviation is similar to that shown in figure 6 . However, in this case the measured error is reduced with a factor of 3 . For low values of the parasitic capacitances good agreement is found between the measurement results depicted in figure 6 and those of figure 5 . 


\subsection{The effect of the reference resistor}

The effect of the reference resistor $R_{\text {ref }}$ on the interface accuracy and resolution was measured with $G_{\mathrm{S}}=$ $49.986 \mu \mathrm{S}$ and a measurement time of $160 \mathrm{~ms}$ (see figure 7). As expected, a larger reference resistance results in a lower standard deviation. However, the maximum measurable conductance is reduced (see figure 5).

\section{Conclusions}

In this paper, an interface for grounded conductivity sensors has been proposed and experimentally evaluated. A high accuracy has been achieved by applying ac square-wave excitation, chopping and auto-calibration. The controllable excitation signal for conductivity sensors enables us to avoid occurrence of electrolysis. The effects of the main nonidealities have been analyzed. The results of these analyses are very useful to optimize the interface system. Depending on the application, the measurement range can be optimized by selecting the reference conductance. Experimental results show that, with a measurement time of about $160 \mathrm{~ms}$, the interface can measure conductance with a standard deviation of about $5.6 \mathrm{nS}$, and a systematic error of $22 \mathrm{nS}$ for a range from $0.01 \mu \mathrm{S}$ to $1 \mathrm{mS}$. The proposed interface is very suitable for implementation in low-cost CMOS technology.

\section{Acknowledgments}

The authors would like to thank Dutch Technology Foundation (STW), The Netherlands, for their financial support on the project DET 6437.

\section{References}

[1] Xu B, Zhang P, Li X and Tao N 2004 Direct conductance measurement of single DNA molecules in aqueous solution Nano Lett. 4 1105-8

[2] Robinson D A, Jones S B, Wraith J M, Or D and Friedman S P 2003 A review of advances in dielectric and electrical conductivity measurement in soils using time domain reflectometry Vadose Zone J. 2 444-75

[3] Thornton Inc. 2000 Product Guide for Process Measurement Instrumentation (USA: Thornton Inc.)

[4] Fougere A J, Brown N L and Hohart E 1992 Integrated CTD Oceanographic Data Collection Platform OCEANOLOGY 92 (Brighton, UK)

[5] Amber Science Inc. 1999 Model 4081 Conductivity Meter (USA: Amber Science Inc.)

[6] Teass H A 1980 Linear ground conductivity measuring system US Patent Appl. No. 4181880

[7] Evans J T, Forgang S and Itskovich G B 1998 Downhole multiphase flow sensor US Patent Appl. No. 5736637

[8] Barben T R 1978 Four electrode conductivity sensor US Patent Appl. No. 641254

[9] Li X and Meijer G C M 2005 A low-cost and accurate interface for four-electrode conductivity sensors IEEE Trans. Instrum. Meas. 54 2433-7

[10] van der Goes F and Meijer G C M 1997 A universal transducer interface for capacitive and resistive sensor elements Analog Integr. Circuits Signal Process. 14 249-60

[11] Gasulla M, Li X and Meijer G C M 2005 The noise performance of a high-speed capacitive-sensor interface based on a relaxation oscillator and a fast counter IEEE Trans. Instrum. Meas. 54 1934-40

[12] Toth F N, Meijer G C M and Kerkvliet H M M 1996 A very accurate measurement system for multielectrode capacitive sensors IEEE Trans. Instrum. Meas. 45 531-5

[13] van der Goes F M L and Meijer G C M 1996 Sigma-delta versus oscillator-based converters in low-cost accurate sensor systems Proc. IMTC96 vol 2 pp 1151-3 